\title{
Clinical features and diagnosis of PFAPA syndrome: approach of the primary care physician
}

\author{
Emiliia Burbela, Liubov Volianska, Oksana Boyarchuk \\ I. Horbachevsky Ternopil National Medical University, Ukraine
}

\section{ABSTRACT}

\begin{abstract}
Introduction: Periodic fever, aphthous stomatitis, pharyngitis and cervical adenitis (PFAPA), described for the first time in 1987 by Marshall, is an inflammatory disease that is characterized by febrile episodes with a specific symptoms. PFAPA syndrome is the most frequent among autoinflammatory diseases. The purpose of this study was to evaluate the clinical features of PFAPA syndrome in children in Western Ukraine and its timely diagnosis by the primary care physicians.

Material and methods: The medical records of 28 children with PFAPA syndrome referred to the inpatient and outpatient departments of Ternopil Regional Children's hospital (tertiary pediatric care hospital) between 2015 and 2020 were reviewed.

Results: The mean age of disease onset was $2.9 \pm 1.5$ years, ranged from 1 to 6 years. The disease occurred more often in male $(71.4 \%, p=0.0013)$. Besides fever, the majority of patients were presented with pharyngitis (100\%), adenitis (64.3\%) and aphtous stomatitis (53.6\%). The effectiveness of steroid drugs occurred in all patients: in 25 (89.3\%) - full response, in $3(10.7 \%)$ - partial response. Tonsillectomy was performed in 2 cases $(7.1 \%)$ and only in one of them PFAPA symptoms have passed. PFAPA syndrome was diagnosed $1.2 \pm 0.5$ years from the onset of the first symptoms, mainly by the specialists of tertiary pediatric care.

Conclusions: The combination of recurrent fever with pharyngitis, adenitis, and aphthous stomatitis may be a clue to the suspicion of PFAPA by primary care physicians who need to seek professional help to rule out other (genetic, metabolic, self-inflammatory, etc.) causes, as this diagnosis is an exclusion diagnosis. There is a need to raise awareness among primary care physicians about PFAPA, which can improve timely diagnosis and treatment, and avoid unnecessary testing and treatment.
\end{abstract}

\section{KEY WORDS:}

PFAPA syndrome, children, diagnosis.

\section{INTRODUCTION}

Periodic fever, aphthous stomatitis, pharyngitis and cervical adenitis (PFAPA), described for the first time in 1987 by Marshall, is an inflammatory disease that is characterized with febrile periodic episodes with a specific symptoms [1-3]. The disease begins more often in healthy children 2-5 years of age and presents with regular (mostly once every 4 or more weeks) episodes of febrile fever in combination with at least one of the following signs: aphthous stomatitis, cervical lymphadenitis, pharyngitis [4-5]. The exacerbation lasts several days, usually 3-6 days [4].

PFAPA syndrome is the most frequent among autoinflammatory diseases. Its prevalence in the world is exactly unknown, however, some publications show that among Norwegian children, the annual incidence is 2.3 per 100 thousand children [6], and Italian researchers indicate 0.4 cases per 1000 children per year [7]. The prevalence of PFAPA syndrome in Ukraine is un-

\section{ADDRESS FOR CORRESPONDENCE:}

Emiliia Burbela, I. Horbachevsky Ternopil National Medical University, Ukraine, e-mail: burbelaei@tdmu.edu.ua 
known due to the lack of a complete registry of such patients, although the prognostic number, according to the number of population, should be at least several thousand cases.

The episodes of fever in patients with PFAPA syndrome may preceded by short-term irritation, fatigue, headache, decreased motor activity, abdominal pain, nausea and diarrhea [8]. Commonly these symptoms occurred 24 hours before the episodes of fever. Children are completely asymptomatic between the episodes of fever and other symptoms $[4,5]$. The disease lasts for several years, passes by adolescence without impairing the physical development of the child. However, in some cases periodic fever remains in adult age or even can have an adult onset [9].

The pathogenesis of PFAPA syndrome remains unknown, although it is now recognized that innate immunity and cytokine dysfunction play a major role. [10-12]. A family history of recurrent fever or recurrent tonsillitis suggests a genetic basis for the syndrome. [3, 8, 9]. Genetic testing for monogenic periodic fever syndromes in 111 patients with PFAPA was negative in 97 of them. [13]. However, the contribution of NLRP 3 and $M E F V$ genes to the etiology of PFAPA disease was previously described [3, 4, 12]; the role of PIK3AP1 and SPON2 was detected recently $[14,15]$.

Oral corticosteroids are the first-line choice to control symptoms, although they do not prevent new episodes [8]. Moreover, after their use interval between flares can become shorter. Tonsillectomy also demonstrated a good result in the majority of cases [16]. If flare episodes are frequent and / or tonsillectomy is ineffective, colchicine may be used to increase the interval between episodes [17].

Thus, the diagnosis of PFAPA syndrome mainly based on clinical criteria [1] and they may differ depending on country, ethnicity due to epigenetic or environmental factors [18].

The purpose of this study was to evaluate the clinical features of PFAPA syndrome in children in Western Ukraine and its timely diagnosis by the primary care physicians.

\section{MATERIAL AND METHODS}

The medical records of 28 children with PFAPA syndrome referred to the inpatient and outpatient departments of Ternopil Regional Children's hospital (tertiary pediatric care hospital) between 2015 and 2020 were reviewed. In total, there were 31 children with unexplained fever. In two during the follow-up examination, cytomegalovirus infection was detected, and in one - acute leukemia. The patients $(n=28)$ were classified as having PFAPA if they fulfilled Thomas diagnostic criteria for PFAPA syndrome [4].
Structured face-to-face interviews with parents and clinical laboratory examinations of children were conducted during hospital visits. The questionnaire for parents provided a complete collection of anamnesis of illness and life and made it possible to reproduce a detailed picture of the disease, as well as its dynamics in each case.

Written consent to the processing and publication of the analysis results was obtained from the parents of all study participants.

The study was conducted in accordance to the basic principles of the Helsinki Declaration of 1975, as revised in 2000.

The data was analyzed using the statistical package STATISTICA 10.0 and Microsoft Excel 2007 to determine the main characteristics (mean, standard deviation). The comparison of frequency parameters was performed using the $\chi^{2}$ test.

\section{RESULTS}

Most of the observed patients were rural residents $(17 / 60.7 \%)$; boys predominated $(20 / 71.4 \%, p=0.0013)$. The age required for inclusion in the study should not exceed 8 years. The average age at the time of the study was $5.9 \pm 1.7$ years, ranged from 2 to 8 years. The mean age at disease onset was $2.9 \pm 1.5$ years, ranged from 1 to 6 years.

The first and the dominant symptom of the disease was fever (Table 1). It was the main reason to refer to medical care. Usually (26/92.9\%) the fever was higher than $38^{\circ} \mathrm{C}$.

The mean duration of the fever episodes was 4.4 \pm 1.0 days, ranged from 2 to 7 days. The fever repeated from every 2-3 weeks to every 2 months.

Symptoms preceding the episodes of fever were reported by only six parents (21.4\%). They include runny nose, cough, arthralgia and weakness. The possibility of any infectious disease was also excluded.

The second frequent symptom was pharyngitis. It occurred in all patients. Cervical lymphadenitis was detected in 11 (39.3\%) children, in one case in combination with an enlargement of the submandibular lymph nodes. We also observed adenoiditis in 7 (25.0\%) patients.

Aphthous stomatitis during the episodes was observed in $15(53.6 \%)$ patients.

Other signs, such as abdominal pain, arthralgia, headache, nausea were less common (Table 1). However, in one patient, fever and pharyngitis were combined with severe abdominal pain, acute appendicitis was diagnosed and urgent surgery was performed.

The physical and mental development of all surveyed matched their age. There were no symptoms related to the disease between episodes of fever. Comorbidities were observed in 8 (28.5\%). Commonly they were presented by biliary tract dysfunction ( 5 patients) and hereditary disorder of connective tissue with skeletal involvement (4 patients). Laboratory data was nonspecific. 
TABLE 1. Symptoms of PFAPA syndrome in the observed children

\begin{tabular}{|l|c|c|}
\hline \multirow{2}{*}{ Symptom } & \multicolumn{2}{|c|}{ Observed children } \\
\cline { 2 - 3 } & $n$ & $\%$ \\
\hline Fever & 28 & 100.0 \\
\hline Recurrence of fever attacks & 28 & 100.0 \\
\hline Pharyngitis & 28 & 100.0 \\
\hline Cervical lymphadenitis & 11 & 39.3 \\
\hline Adenoiditis & 7 & 25.0 \\
\hline Aphthous stomatitis & 15 & 53.6 \\
\hline Abdominal pain & 5 & 17.6 \\
\hline Arthralgia & 3 & 10.7 \\
\hline Headache & 6 & 21.4 \\
\hline Nausea & 3 & 10.7 \\
\hline
\end{tabular}

Clinical and laboratory examination allows to exclude other diseases with periodic fever, such as cyclic neutropenia, autoinflammatory and autoimmunity disorders, viral and bacterial infections.

One boy was observed to have an increase in episodes of fever with pharyngitis up to 1 time in 2 weeks after Epstein-Barr virus infection, that was confirmed by serological testing. Autoinflammatory diseases were also suspected, and sequence analysis identified variant of uncertain significance in NLRC4 gene that is associated with autosomal dominant familial cold autoinflammatory syndrome and autosomal dominant macrophage activation syndrome.

Recurrent fever with tonsillitis/pharyngitis or tonsillectomy in parents was reported in 7 (25\%) cases.

PFAPA syndrome was diagnosed $1.2 \pm 0.5$ years from the onset of the first symptoms. All patients were referred by primary care physicians to the tertiary pediatric care hospital where diagnosis was set, only in 4 (14.4\%) cases patients were referred with suspected PFAPA syndrome. In $20(71.4 \%)$ PFAPA syndrome was diagnosed by pediatric immunologists, in rest cases - by other specialists (otolaryngologist, infectious disease specialist, and rheumatologist).

The majority of parents reported about partial efficiency $(10 / 35.7 \%)$ or complete inefficiency (9/32.1\%) of antipyretic drugs (Ibuprofen in a single dose of $10 \mathrm{mg} / \mathrm{kg}$ three times a day or paracetamol in a single dose of $12 \mathrm{mg} / \mathrm{kg}$ three times a day). In 5 (17.9\%) cases, the effectiveness of antipyretic drugs ranged from resistance to a tendency to decrease in fever, depending on each exacerbation, and only in $4(14.3 \%)$ cases, parents reported full effectiveness. Instead, the effectiveness of steroid drugs occurred in all patients, however, in 25 (89.3\%) we observed full efficacy and in 3 (10.7\%) - partial response. Glucocorticoids at a dose of $1 \mathrm{mg} / \mathrm{kg}$ for prednisolone mostly interrupted the cascade of clinical symptoms. However, 15 (53.6\%) observed children needed the second doses of glucocorticoids.
Tonsillectomy was performed in 2 cases (7.1\%), and only in one of them the symptoms of PFAPA were eliminated. An adenoidectomy was performed in 3 children and mothers reported that flares became less frequent after this surgery.

\section{DISCUSSION}

The diagnosis of PFAPA is based mainly on clinical criteria [1]. There are no specific laboratory findings to confirm diagnosis [19]. On the other hand, PFAPA is a diagnosis of exclusion, therefore other causes of recurrent fever should be ruled out [19].

Fever of infectious or noninfectious genesis is one of the most common causes to seek for medical advice in preschool children. Mostly fever is caused by the upper respiratory tract infections, and usually is accompanied by regional lymphadenitis, and sometimes by aphthous rash on the oral mucosa. Therefore, primary care physicians treated first episodes of PFAPA as an acute respiratory disease and, in its recurrence, as a relapse.

Cluster recurrence of symptoms leaded to unnecessary laboratory and instrumental examinations, multiple consultations by doctors of related specialties, such as: otolaryngologist, dentist, hematologist, rheumatologist, infectious disease specialist, immunologist. Accordingly this leads to polypragmasia. As recurrent infections are the main signs of primary immunodeficiency [20], these patients often were referred to the immunologist, and as a result the PFAPA syndrome in our study mostly was diagnosed by the immunologists (71.4\%). The small number of cases suspected of PFAPA by primary care physicians, despite all criteria for diagnosis and the delay in diagnosis, indicates a need for increased awareness of this syndrome, especially among primary care physicians [21, 22]. According to the PFAPA criteria, the first manifestations are determined before 5 years of age [5]. In children from our study, the average age also ranged $2.9 \pm 1.5$ years. But in $7.1 \%(n=2)$ children the first manifestations were recorded at 5.5 years and 6 years. This was also noted in the Hofer et al. study (2014), where they reported that $10.3 \%$ of the cases had late onset [13].

The incomplete cluster of PFAPA obviously affects the timeliness of diagnosis and makes the average age of diseases onset variable according to the data of various studies $[9,23]$.

Multicentre study revealed that the classic combination of symptoms of PFAPA is more common in boys [13] that was also observed in our study.

Only $21.4 \%$ of patients had predictors of PFAPA flares, however, other studies reported them in the vast majority of cases and noted variability of additional symptoms in their patients $[13,24]$. A quarter of the children in our study had adenoiditis, although other studies did not allocate adenoiditis separately, but in some studies both tonsillectomy and adenoidectomy were performed [25]. 
The efficacy of steroid therapy (complete in $89.3 \%$ of cases) is in accordance with EUROFEVER registry data, which confirms the widespread use of steroids for disease outbreaks with $90 \%$ efficacy in those receiving treatment [24]. However, in another study, rapid cancellation of a fever episode after a single dose of steroids was observed only in $63 \%$ of patients, whereas $32 \%$ found partial response and 5\% did not respond at all [13].

The need of tonsillectomy in patients with PFAPA syndrome is disputable [19]. It was performed in two of our patients $(7.1 \%)$, and only in one of them it was successful, although other publications reported more frequent use of this method of treatment (45-93\% of patients) with good results $[3,6,16,26]$.

However, genetic testing was performed in our study only in one case, it identified variant of uncertain significance in NLRC4 gene, that need further investigation of its role in autoinflammation, as even carries of $M E F V$ and NLRP3 genes were susceptibile to autoinflammation [3].

Thus, all children in our study had a complete cluster of PFAPA syndrome with a frequency of individual symptoms similar to those as reported by other researches [3, $6,8]$. In all observed patients, the reaction of lymphoid tissue was combined in time with the onset of fever, in which only $14.3 \%$ of patients responded to the use of antipyretics. The effectiveness of steroid drugs occurred in all patients. A good response to the steroids can be used as an additional criterion to confirm the disease along with generally accepted clinical criteria. Tonsillectomy is not as popular strategy for treating the PFAPA syndrome among our physicians as in other populations, which may be due to a lack of knowledge among otolaryngologists about its effectiveness and the disagreement of parents who know about the favorable course of the syndrome and its resolution with age. Noteworthy is the use of the alternative colchicine cimetidine or anakinra to prevent recurrent episodes of the disease [27].

Delayed establishment of this diagnosis after 1.2 \pm 0.5 years from the appearance of the first symptoms in the presence of classic cluster reproduction of the symptom complex reflected the need to increase the professional level of doctors of the first contact.

\section{CONCLUSIONS}

In the cases of recurrent fever that is accompanied by pharyngitis, adenitis, and aphthous stomatitis primary care physicians should suspect PFAPA syndrome and refer to a specialist to exclude other (genetic, metabolic, auto-ignition, etc.) reasons, since this diagnosis is a diagnosis of exclusion. The diagnosis of PFAPA requires a longer observation of the patient and the use of special laboratory tests, including genetic ones. Corticosteroids are an effective treatment strategy and its efficacy can be used as additional criterion to diagnose PFAPA syndrome.
Raising awareness of PFAPA among primary care physicians can improve timely diagnosis and treatment by avoiding unnecessary testing and treatment.

\section{DISCLOSURE}

The authors declare no conflict of interest.

\section{REFERENCES}

1. Marshall GS, Edwards KM, Butler J, Lawton AR. Syndrome of periodic fever, pharyngitis, and aphtous stomatitis. J Pediatr 1987; 110: 43-46.

2. Batu ED. Periodic fever, aphthous stomatitis, pharyngitis, and cervical adenitis (PFAPA) syndrome: main features and an algorithm for clinical practice. Rheumatol Int 2019; 39: 957-970.

3. Perko D, Debeljak M, Toplak N, et al. Clinical features and genetic background of the periodic Fever syndrome with aphthous stomatitis, pharyngitis, and adenitis: a single center longitudinal study of 81 patients. Mediators Inflamm 2015; 2015: 293417.

4. Vanoni F, Theodoropoulou K, Hofer M. PFAPA syndrome: a review on treatment and outcome. Pediatr Rheumatol Online J 2016; 14: 38.

5. Thomas KT, Feder HM Jr, Lawton AR, et al. Periodic fever syndrome in children. J Pediatr 1999; 135: 15-21.

6. Førsvoll J, Kristoffersen EK, Øymar K. Incidence, clinical characteristics and outcome in Norwegian children with periodic fever, aphthous stomatitis, pharyngitis and cervical adenitis syndrome; a population-based study. Acta Paediatr 2013; 102: 187-192.

7. Barbi E, Besoli G, Brusadin L, et al. Incidence and natural history of PFAPA in Friuli-Venezia Giulia: a collaborative study by family paediatricians. Medico e Bambino 2001; 20: 234-238.

8. Foster KJ, RodriguesJ, Lee J, et al. Periodic Fever, Aphthous Stomatitis, Pharyngitis, Adenitis Syndrome at a Single Children's Hospital. Int J Aller Medications 2018; 4: 034.

9. Hernández-Rodríguez J, Ruíz-Ortiz E, Tomé A, et al. Clinical and genetic characterization of the autoinflammatory diseases diagnosed in an adult reference center. Autoimmun Rev 2016; 15: 9-15.

10. Stojanov S, Hoffmann F, Kery A, et al. Cytokine profile in PFAPA syndrome suggests continuous inflammation and reduced antiinflammatory response. Eur Cytokine Netw 2006; 17: 90-97.

11. Stojanov S, Lapidusa S, Chitkara P, et al. Periodic fever, aphthous stomatitis, pharyngitis, and adenitis (PFAPA) is a disorder of innate immunity and Th1 activation responsive to IL-1 blockade. Proc Natl Acad Sci U S A 2011; 108: 7146-7153.

12. Kolly L, Busso N, Von Scheven-Gete A, et al. Periodic fever, aphthous stomatitis, pharyngitis, cervical adenitis syndrome is linked to dysregulated monocyte IL- $1 \beta$ production. J Allergy Clin Immunol 2013; 131: 1635-1643.

13. Hofer M, Pillet $\mathrm{P}$, Cochard M-M, et al. International periodic fever, aphthous stomatitis, pharyngitis, cervical adenitis syndrome cohort: description of distinct phenotypes in 301 patients. Rheumatology (Oxford) 2014; 53: 1125-1129.

14. Lovšin E, Kovač J, Tesovnik T, et al. PIK3AP1 and SPON2 Genes Are Differentially Methylated in Patients With Periodic Fever, Aphthous Stomatitis, Pharyngitis, and Adenitis (PFAPA) Syndrome. Front Immunol 2020; 11: 1322.

15. Manthiram K, Preite S, Dedeoglu F, et al. Common genetic susceptibility loci link PFAPA syndrome, Behçet's disease, and recurrent aphthous stomatitis. Proc Natl Acad Sci U S A 2020; 117: 14405-14411.

16. Aktas O, Aytuluk HG, Caliskan SK, et al. Long-term follow-up of tonsillectomy efficacy in children with PFAPA syndrome. Braz J Otorhinolaryngol 2019; 85: 78-82. 
17. Tasher D, Stein M, Dalal I, et al. Colchicine prophylaxis for frequent periodic fever, aphthous stomatitis, pharyngitis and adenitis episodes. Acta Paediatr 2008; 97: 1090-1092.

18. Batu ED, Kara Eroğlu F, Tsoukas P, et al. Periodic Fever, Aphthosis, Pharyngitis, and Adenitis Syndrome: Analysis of Patients From Two Geographic Areas. Arthritis Care Res (Hoboken) 2016; 68: 1859-1865.

19. Terreri MT, Bernardo WM, Len CA, et al. Guidelines for the management and treatment of periodic fever syndromes: periodic fever, aphthous stomatitis, pharyngitis and adenitis syndrome. Rev Bras Reumatol Engl Ed 2016; 56: 52-57.

20. Boyarchuk O, Dmytrash L. Clinical Manifestations in the Patients with Primary Immunodeficiencies: Data from One Regional Center. Turk J Immunol 2019; 17: 113-119.

21. Hariyan T, Kinash M, Kovalenko R, et al. Evaluation of awareness about primary immunodeficiencies among physicians before and after implementation of the educational program: A longitudinal study. PLoS ONE 2020; 15: e0233342.

22. Boyarchuk O, Volyanska L, Kosovska T, et al. Awareness about primary immunodeficiency diseases among medical students. Georgian Med News 2018; 12: 124-130.

23. Cattalini M, Soliani M, Rigante D, et al. Basic Characteristics of Adults with Periodic Fever, Aphthous Stomatitis, Pharyngitis, and Adenopathy Syndrome in Comparison with the Typical Pediatric Expression of Disease. Mediators Inflamm 2015; 2015: 570418.

24. Ter Haar N, Lachmann H, Özen S, et al. Treatment of autoinflammatory diseases: results from the Eurofever Registry and a literature review. Ann Rheum Dis 2013; 72: 678-685.

25. Garavello W, Romagnoli M, Gaini RM. Effectiveness of adenotonsillectomy in PFAPA syndrome: a randomized study. J Pediatr 2009; 155: 250-253

26. Perko D, Debeljak M, Toplak N, Avčin T. Clinical Features and Genetic Background of the Periodic Fever Syndrome with Aphthous Stomatitis, Pharyngitis, and Adenitis: A Single Center Longitudinal Study of 81 Patients. Mediators Inflamm 2015; 2015: 293417.

27. Gaggiano C, Rigante D, Sota J, et al. Treatment options for periodic fever, aphthous stomatitis, pharyngitis, and cervical adenitis (PFAPA) syndrome in children and adults: a narrative review. Clin Rheumatol 2019; 38: 11-17. 